\title{
ON THE APPROXIMATION OF FIXED POINTS FOR LOCALLY PSEUDO-CONTRACTIVE MAPPINGS
}

\author{
CLAUDIO H. MORALES AND SIMBA A. MUTANGADURA
}

(Communicated by Palle E. T. Jorgensen)

\begin{abstract}
Let $X$ and its dual $X^{*}$ be uniformly convex Banach spaces, $D$ an open and bounded subset of $X, T$ a continuous and pseudo-contractive mapping defined on $\operatorname{cl}(D)$ and taking values in $X$. If $T$ satisfies the following condition: there exists $z \in D$ such that $\|z-T z\|<\|x-T x\|$ for all $x$ on the boundary of $D$, then the trajectory $t \rightarrow z_{t} \in D, t \in[0,1)$, defined by $z_{t}=t T\left(z_{t}\right)+(1-t) z$ is continuous and converges strongly to a fixed point of $T$ as $t \rightarrow 1^{-}$.
\end{abstract}

\section{INTRODUCTION}

Let $X$ be a real Banach space, and let $D$ be a subset of $X$. An operator $T: D \rightarrow X$ is said to be $k$-pseudo-contractive $(k>0)$ (see [9]) if for each $x$, $y \in D$ and $\lambda>k$

$$
(\lambda-k)\|x-y\| \leq\|\lambda(x-y)-(T x-T y)\| .
$$

For $k=1 \quad(k<1)$ such mappings are said to be pseudo-contractive (respectively, strongly pseudo-contractive). By letting $r=1 /(\lambda-1)$ and $k=1$ in (1), we may derive the original definition of pseudo-contractive mappings, due to Browder [1], as follows:

$$
\|x-y\| \leq\|(1+r)(x-y)-r(T x-T y)\|
$$

holds for all $x, y \in D$ and all $r>0$. However, by taking a semi-inner product approach (see also Kato [6]) we may describe (2) by

$$
\langle T x-T y, j\rangle \leq\|x-y\|^{2}
$$

for some $j \in J(x-y)$. The mapping $J: X \rightarrow 2^{X^{*}}$ is called the normalized duality mapping which is defined by

$$
J(x)=\left\{j \in X^{*}:\langle x, j\rangle=\|x\|^{2},\|j\|=\|x\|\right\} .
$$

Here $\langle\cdot, \cdot\rangle$ denotes the generalized duality pairing. We should mention that this latter family of mappings is intimately related to the so-called accretive operators, which play an important role in the theory of evolution equations.

Received by the editors December 10, 1992 and, in revised form, May 3, 1993.

1991 Mathematics Subject Classification. Primary 47H10.

Key words and phrases. Pseudo-contractive mappings, uniform convexity. 
Furthermore, if condition (1) holds locally, i.e., if each point $x \in D$ has a neighborhood $U$ such that the restriction of $T$ to $U$ is $k$-pseudo-contractive with (uniform) constant $k$, then $T$ is said to be locally $k$-pseudo-contractive.

The purpose of this paper is to continue the discussion concerning the strong convergence of the path $t \rightarrow z_{t}, 0 \leq t<1$, defined by (4). In fact, we prove for locally pseudo-contractive mappings under condition (3) that the strong $\lim _{t \rightarrow 1^{-}} z_{t}$ exists and is a fixed point of $T$. We should also mention that this result appears to be new even in Hilbert spaces. The first results of this nature were established by Browder [2] and Browder and Petryshyn [3], and more recently Bruck et al. [4] proved Theorem 1 for locally nonexpansive mappings. Another result, perhaps more revealing, is Proposition 2 where we prove that the mapping $2 I-T$ is globally one-to-one. This fact, by itself, appears to have a significant connotation in the theory of locally pseudo-contractive mappings.

To fix our notation, we will denote the closure and boundary of $D$ by $\bar{D}$ and $\partial D$ respectively, and for $u, v \in X$ we use $\operatorname{seg}[u, v]$ to denote the segment $\{t u+(1-t) v: t \in[0,1]\}$. We will also use $B(x ; r)$ and $\bar{B}(x ; r)$ to stand for the open ball $\{z \in X:\|x-z\|<r\}$ and the closed ball $\{z \in X:\|x-z\| \leq r\}$ respectively. We denote the distance between the sets $A$ and $B$ by $\operatorname{dist}(A, B)$, i.e.,

$$
\operatorname{dist}(A, B)=\inf \{\|a-b\|: a \in A, b \in B\}
$$

\section{PRELIMINARIES}

The main objective of this paper is to extend Theorem 1 of Morales [11]. We begin by stating this result under the following proposition.

Proposition 1 ([11]). Let $X$ and $X^{*}$ be uniformly convex Banach spaces, let $D$ be a bounded open subset of $X$, and let $T: \bar{D} \rightarrow X$ be a uniformly continuous mapping which is locally pseudo-contractive on $D$. Suppose there exists $z \in D$ such that

$$
\|z-T z\|<\|x-T x\| \quad \text { for all } x \in \partial D .
$$

Then there exists a unique path $t \rightarrow z_{t} \in D, t \in[0,1)$, satisfying

$$
z_{t}=t T\left(z_{t}\right)+(1-t) z
$$

where the strong $\lim _{t \rightarrow 1^{-}} z_{t}$ exists, and this limit is a fuxed point for $T$.

As a consequence of the proof of this previous result, the following can easily be derived.

Corollary 1. Let $X$ and $X^{*}$ be uniformly convex Banach spaces, let $D$ be a bounded open subset of $X$, and let $T: \bar{D} \rightarrow X$ be a continuous mapping which is locally pseudo-contractive on $D$. Suppose there exists $z \in D$ such that (3) holds. Then there exists a unique path $t \rightarrow z_{t} \in D, t \in[0,1)$, satisfying (4). If, in addition, this path $\left\{z_{t}: 0 \leq t<1\right\}$ satisfies

$$
\operatorname{dist}\left(\left\{z_{t}\right\}, \partial D\right)>0 \text {, }
$$

then the strong $\lim _{t \rightarrow 1^{-}} z_{t}$ exists, and this limit is a fixed point of $T$.

In view of Corollary 1 , we should observe that uniform continuity is essential to prove condition $(*)$. On the other hand, due to the well-known fact that 
every locally nonexpansive mapping is globally nonexpansive on convex sets, condition $(*)$ can also be shown (see [7]). This fact allows us to derive the following special case, which was obtained earlier by Bruck et al. [4].

Corollary 2. Let $X$ and $X^{*}$ be uniformly convex Banach spaces, let $D$ be an open subset of $X$, and let $T: \bar{D} \rightarrow X$ be a continuous mapping which is locally nonexpansive on $D$. Suppose (3) holds for some $z \in D$. Then there exists a unique path $t \rightarrow z_{t} \in D, t \in[0,1)$, satisfying (4). If, in addition, this path $\left\{z_{t}: 0 \leq t<1\right\}$ is bounded, then the strong $\lim _{t \rightarrow 1^{-}} z_{t}$ exists, and this limit is a fixed point of $T$.

\section{MAIN RESULT}

Now we are ready to extend Proposition 1 by replacing the uniform continuity by mere continuity. In fact, this process will take place by reformulating the original problem into a problem involving locally nonexpansive mappings. However, using this argument, we will lose some properties which will not allow us to use the result of [11].

Theorem 1. Let $X$ and $X^{*}$ be uniformly convex Banach spaces, let $D$ be a bounded open set of $X$, and let $T: \bar{D} \rightarrow X$ be a continuous mapping which is locally pseudo-contractive on $D$. Suppose there exists $z \in D$ such that

$$
\|z-T z\|<\|x-T x\| \quad \text { for all } x \in \partial D .
$$

Then there exists a unique path $t \rightarrow z_{t} \in D, t \in[0,1)$, satisfying

$$
z_{t}=t T z_{t}+(1-t) z
$$

where the strong $\lim _{t \rightarrow 1^{-}} z_{t}$ exists, and this limit is a fixed point of $T$.

Before proving this result, we need to introduce some basic facts that will be used in the proof of the main theorem. We begin with a lemma, whose proof can be found in Kirk [7]. It might be worthwhile to mention that the existence of the path $t \rightarrow z_{t}$ was previously established by Kirk and Morales [8] for general Banach spaces. Therefore, it is the strong convergence of this path which is actually at stake.

Lemma 1 (cf. Fact II of [7]). Let $D$ be an open subset of a Banach space $X$, and suppose $F: \bar{D} \rightarrow X$ is a continuous mapping which is locally strongly accretive on $D$. Let $u=F(x), x \in D$, and let $S=\operatorname{seg}[u, v]$ such that $\operatorname{seg}[u, v) \subset$ $F(D)$ for some $v \in X$. Then $v \in F(\bar{D})$ and there exists a unique path (up to parametrization) whose image $\Gamma$ begins at $x$, ends at a point $w \in F^{-1}(v)$, and for which $F(\Gamma)=S$. Moreover, the inverse of the restriction of $F$ to $\Gamma$ is a Lipschitz mapping of $S$ onto $\Gamma$.

Our next lemma can also be found in Kirk [7]. For complete details of its proof, see pages 94 and 97 of [7] respectively.

Lemma 2. Let $X$ be a Banach space, and let $T: \bar{D} \rightarrow X$ be as in Theorem 1, satisfying (3) for some $z \in D$. Suppose $F x=2 x-T x$. Then:

(i) $\operatorname{seg}[z, F z] \subset F(D)$.

(ii) Let $x \in D$ such that $\|x-T x\|<\rho=\|z-T z\| / 3$.

Then $B(F x ; \rho) \subset F(D)$. 
Proposition 2. Let $X$ be a Banach space, let $D$ be a connected open subset of $X$, and let $T: \bar{D} \rightarrow X$ be a continuous mapping which is locally pseudo-contractive on $D$. Then the mapping $F x=2 x-T x$ is globally one-to-one on $D$.

Proof. We first observe that $F$ is continuous on $\bar{D}$ and locally strongly accretive on $D$ and, thus, locally expansive on $D$. This means, for each $x \in D$, there exists a neighborhood $U$ such that for $u, v \in U$

$$
\|u-v\| \leq\|F u-F v\| .
$$

Also, as a consequence of Deimling's domain invariance theorem [5, Theorem 3], $F$ maps open subsets of $D$ onto open sets of $X$. Now we are ready to show that $F$ is a one-to-one mapping on $D$. To see this, let $y \in F(D)$. Since $F(D)$ is open, there exists $v \in F(D)$ so that $\operatorname{seg}[v, y] \subset F(D)$. Choose $u \in D$ such that $v=F u$. Then, by Lemma 1 , there exists a unique path $\gamma:[0,1] \rightarrow D$ so that $\gamma(0)=u, \gamma(1)=x$ for some $x \in F^{-1}(y)$, and for which $F(\gamma([0,1]))=\operatorname{seg}[v, y]$. Suppose there is $x_{1} \in D$ such that $x_{1} \neq x$ and $x_{1} \in F^{-1}(y)$. Let $B\left(x_{1} ; \eta\right) \subset D$ for some $\eta>0$. Then there exists $\varepsilon>0$ for which $B(y ; \varepsilon) \subset F\left(B\left(x_{1} ; \eta\right)\right)$. On the other hand, due to the continuity of $F$ at $x$, there exists $\delta>0$ such that $F(B(x ; \delta)) \subset B(y ; \varepsilon)$. Now, if we consider the restriction of $F$ to $\widetilde{D}=D \backslash \bar{B}(x ; \delta / 2)$, it follows that $\operatorname{seg}[v, y] \subset F(\widetilde{D})$. Once again, there exists a (unique) path $\gamma_{1}:[0,1] \rightarrow \widetilde{D}$ such that $\gamma_{1}(0)=u$, $\gamma_{1}(1)=x_{2}$ for some $x_{2} \neq x$, and for which $F\left(\gamma_{1}[0,1]\right)=\operatorname{seg}[v, y]$. This contradicts the uniqueness of the path $\gamma$. This implies $F^{-1}(y)=\{x\}$, and hence $F$ is a homeomorphism from $D$ onto $F(D)$.

Proof of Theorem 1. In view of Proposition 2, the mapping $F$ is not necessarily one-to-one on $\bar{D}$. Therefore, we will redefine the domain of $T$ to assure that $F$ is also invertible on the boundary of its domain. Due to Theorem 2 of [10], we may select $w \in D$ such that

$$
\|w-T w\|<\|z-T z\| .
$$

We now replace $D$ by the open set $D_{0}$ defined by

$$
D_{0}=\{x \in D:\|x-T x\|<\|z-T z\|\} .
$$

Then $\partial D_{0} \subset D$ and

$$
\|w-T w\|<\|x-T x\| \quad \text { for } x \in \partial D_{0} .
$$

This means the path $t \rightarrow w_{t}$ for which $w$ satisfies (5) exists and is uniquely defined on $[0,1)$ (see Lemma 3 of [11]). By Lemma 2(i), we know that $\operatorname{seg}[w, F w] \subset F\left(D_{0}\right)$, and since by Proposition $2 F^{-1}$ exists on $F\left(D_{0}\right)$, we derive that $F^{-1}$ is nonexpansive on $\operatorname{seg}[w, F w]$ and

$$
\left\|w-F^{-1}(w)\right\| \leq\|w-F(w)\|<\|x-T x\|
$$

for all $x \in \partial D_{0}$. Due to the fact $\partial F\left(D_{0}\right)=F\left(\partial D_{0}\right)$, we may say that for each $y \in \partial F\left(D_{0}\right)$, there exists $x \in \partial D_{0}$ such that $y=F x$ and

$$
\left\|w-F^{-1}(w)\right\|<\left\|y-F^{-1}(y)\right\| .
$$


Consequently, by Corollary 2, there exists a unique path $t \rightarrow u_{t} \in F\left(D_{0}\right)$, $t \in[0,1)$, satisfying the equation

$$
u_{t}=t F^{-1}\left(u_{t}\right)+(1-t) w
$$

where the $\lim _{t \rightarrow 1^{-}} u_{t}$ exists and is a fixed point of $F^{-1}$. Due to uniqueness of the path $t \rightarrow w_{t}, F^{-1}\left(u_{t}\right)=w_{s}$ where $s=1 /(2-t)$, and hence the strong $\lim _{t \rightarrow 1^{-}} w_{t}$ exists. Since this limit exists for every $w \in D_{0}$ that satisfies (5), we choose a sequence $\left\{z^{n}\right\}$ in $D_{0}$ such that $z^{n} \rightarrow z$. For each $z^{n}$, the corresponding path can be written as

$$
z_{t}^{n}=t T\left(z_{t}^{n}\right)+(1-t) z^{n}, \quad t \in[0,1] .
$$

Let $\eta>0$ such that $B(z ; \eta) \subset D$, and let $k \in \mathbb{N}$ such that $z^{n} \in B(z ; \eta / 4)$ for all $n \geq k$. From (6) and the fact that each $z_{t}^{n} \in D_{0}$, we have

$$
\left\|z_{t}^{n}-z^{n}\right\|=\frac{t}{1-t}\left\|z_{t}^{n}-T\left(z_{t}^{n}\right)\right\| \leq \frac{t}{1-t}\|z-T z\| .
$$

Then there exists $t_{0} \in(0,1)$ for which $\left\|z_{t}^{n}-z^{n}\right\|<\eta / 4$ for $t \in\left[0, t_{0}\right]$ and $n \in \mathbb{N}$. This implies that $z_{t}^{n} \in B(z ; \eta / 2)$ for all $t \in\left[0, t_{0}\right]$ and for all $n \geq k$. Since $T$ is globally pseudo-contractive on $B(z ; \eta)$, there exists $j \in J\left(z_{t}^{n}-z_{t}^{m}\right)$ so that

$$
\begin{aligned}
\left\langle z_{t}^{n}-z_{t}^{m}, j\right\rangle & =t\left\langle T z_{t}^{n}-T z_{t}^{m}, j\right\rangle+(1-t)\left\langle z^{n}-z^{m}, j\right\rangle \\
& \leq t\left\|z_{t}^{n}-z_{t}^{m}\right\|^{2}+(1-t)\left\langle z^{n}-z^{m}, j\right\rangle
\end{aligned}
$$

for $n, m \geq k$ and $t \in\left[0, t_{0}\right]$. Then we may obtain that

$$
\left\|z_{t}^{n}-z_{t}^{m}\right\| \leq\left\|z^{n}-z^{m}\right\| \quad \text { for all } m, n \geq k \text { and } t \in\left[0, t_{0}\right] .
$$

This means the sequence $\left\{z_{t}^{n}\right\}_{n=1}^{\infty}$ is a convergent sequence for each $t \in\left[0, t_{0}\right]$, say, $\lim _{n \rightarrow \infty} z_{t}^{n}=\tilde{z}_{t}$. Therefore, by (6) we have

$$
\tilde{z}_{t}=t T\left(\tilde{z}_{t}\right)+(1-t) z \quad \text { for } t \in\left[0, t_{0}\right] \text {. }
$$

Once again due to uniqueness of the path, $\tilde{z}_{t}=z_{t}$ for all $t$ for which $\left\{z_{t}^{n}\right\}$ is convergent. We now define the set

$$
\begin{aligned}
& E=\left\{s \in[0,1]:\left\|z_{t}^{n}-z_{t}^{m}\right\| \leq\left\|z^{n}-z^{m}\right\|\right. \text { for all } \\
& \left.\qquad t \in[0, s], n, m \geq n_{s}, \text { for some } n_{s} \in \mathbb{N}\right\} .
\end{aligned}
$$

Since $t_{0} \in E$ and $z_{t_{0}} \in D$, there exist $\delta>0$ and $l \in \mathbb{N}$ such that $B\left(z_{t_{0}} ; \delta\right) \subset D$ and

$$
\left\|z_{t_{0}}^{n}-z_{t_{0}}\right\|<\delta / 5 \quad \text { for all } n \geq l \text {. }
$$

Hence there exists $\alpha>0$ for which

$$
z_{t}^{l} \in B\left(z_{t_{0}} ; \delta / 4\right) \quad \text { for all } t \in\left(t_{0}-\alpha, t_{0}+\alpha\right) .
$$

This implies that $\left\|z_{t}^{n}-z_{t_{0}}\right\|<\delta / 2$ for all $n \geq l$ and $t \in\left(t_{0}-\alpha, t_{0}+\alpha\right)$. Otherwise, we may choose $j \geq l$ and $t_{1} \in\left(t_{0}-\alpha, t_{0}+\alpha\right)$ so that $\left\|z_{t_{1}}^{j}-z_{t_{0}}\right\|=$ $\delta / 2$. This implies $z_{t_{1}}^{l}, z_{t_{1}}^{j} \in B\left(t_{t_{0}} ; \delta\right)$, and since $T$ is pseudo-contractive on $B\left(z_{t_{0}} ; \delta\right)$, we obtain

$$
\left\|z_{t_{1}}^{l}-z_{t_{1}}^{j}\right\| \leq\left\|z^{l}-z^{j}\right\|<\delta / 5 .
$$

This is a contradiction, since $\left\|z_{t_{1}}^{l}-z_{t_{1}}^{j}\right\| \geq \delta / 4$. Therefore, $\left(t_{0}-\alpha, t_{0}+\alpha\right) \subset E$. Due to the continuity of the path $t \rightarrow z_{t}^{n}$, we deduce that $t_{0}+\alpha \in E$. This 
means $[0,1) \subset E$. It remains to show that $1 \in E$. To see this, we first mention that

$$
\left\|z_{t}^{n}-T\left(z_{t}^{n}\right)\right\|=\left(\frac{1}{t}-1\right)\left\|z^{n}-z_{t}^{n}\right\| .
$$

Since $D$ is bounded, there exists $s \in(0,1)$ such that

$$
\left\|z_{t}^{n}-T\left(z_{t}^{n}\right)\right\| \leq \rho \quad \text { for all } n \geq 1 \text { and } t \in[s, 1] .
$$

Therefore, by Lemma 2(ii), $B\left(F\left(z_{t}^{n}\right) ; \rho\right) \subset F(D)$. We choose $k \in \mathbb{N}$ such that $k \geq n_{s}$ and $\left\|z^{n}-z^{m}\right\|<\rho$ for all $n, m \geq k$. Then

$$
\left\|F\left(z_{t}^{n}\right)-F\left(z_{t}^{m}\right)\right\| \leq\left\|z^{n}-z^{m}\right\| \quad \text { for } n, m \geq k \text { and } t \in[s, 1] .
$$

Otherwise, we may find $i, j \geq k$ and $r \in(s, 1)$ such that $\left\|F\left(z_{r}^{i}\right)-F\left(z_{r}^{j}\right)\right\|>$ $\left\|z^{i}-z^{j}\right\|$, while $\left\|F\left(z_{s}^{i}\right)-F\left(z_{s}^{j}\right)\right\| \leq\left\|z^{i}-z^{j}\right\|$. Then there exists $t \in[s, r]$ such that

$$
\left\|z^{i}-z^{j}\right\|<\left\|F\left(z_{t}^{i}\right)-F\left(z_{t}^{j}\right)\right\|<\rho .
$$

Hence $\operatorname{seg}\left[F\left(z_{t}^{i}\right), F\left(z_{t}^{j}\right)\right] \subset F(D)$, and thus $\left\|z_{t}^{i}-z_{t}^{j}\right\| \leq\left\|F\left(z_{t}^{i}\right)-F\left(z_{i}^{j}\right)\right\|$. Since

$$
F\left(z_{t}^{i}\right)-F\left(z_{t}^{j}\right)=\left(2-\frac{1}{t}\right)\left(z_{t}^{i}-z_{t}^{j}\right)+\left(\frac{1}{t}-1\right)\left(z^{i}-z^{j}\right)
$$

we derive that $\left\|F\left(z_{t}^{i}\right)-F\left(z_{t}^{j}\right)\right\| \leq\left\|z^{i}-z^{j}\right\|$. This contradicts (7). Therefore,

$$
\left\|z_{t}^{n}-z_{t}^{m}\right\| \leq\left\|z^{n}-z^{m}\right\| \quad \text { for } n, m \geq k \text { and } t \in[s, 1] .
$$

This implies $1 \in E$, and hence $E=[0,1]$. This means there exists $n_{0} \in \mathbb{N}$ so that

$$
\left\|z_{t}^{n}-z_{t}^{m}\right\| \leq\left\|z^{n}-z^{m}\right\| \quad \text { for all } n, m \geq n_{0} \text { and } t \in[0,1] .
$$

In particular, $\left\{z_{1}^{n}\right\}$ is a convergent sequence, say, to $z_{1}$. Then for $\varepsilon>0,(8)$ implies there exists $n \in \mathbb{N}$ such that $\left\|z_{t}^{n}-z_{t}\right\|<\varepsilon / 3$ for all $t \in[0,1]$. Also, we may choose $\delta>0$ satisfying

$$
\left\|z_{t}^{n}-z_{1}^{n}\right\|<\varepsilon / 3 \quad \text { for all } t \in(1-\delta, 1] .
$$

Therefore,

$$
\begin{aligned}
\left\|z_{t}-z_{1}\right\| & \leq\left\|z_{t}-z_{t}^{n}\right\|+\left\|z_{t}^{n}-z_{1}^{n}\right\|+\left\|z_{1}^{n}-z_{1}\right\| \\
& <\varepsilon / 3+\varepsilon / 3+\varepsilon / 3=\varepsilon
\end{aligned}
$$

for $t \in(1-\delta, 1]$. This completes the proof.

\section{ACKNOWLEDGMENT}

This manuscript was prepared while the second author was visiting the Alabama A \& M University, and he would like to thank Professor Jerry Shipman for his hospitality in the Department of Mathematics. The authors wish to thank the referee for his valuable comments. 


\section{REFERENCES}

1. F. E. Browder, Nonlinear mappings of nonexpansive and accretive type in Banach spaces, Bull. Amer. Math. Soc. 73 (1967), 875-882.

2. __ Convergence of approximants to fixed points of nonexpansive nonlinear mappings in Banach spaces, Arch. Rational Mech. Anal. 24 (1967), 82-90.

3. F. E. Browder and W. V. Petryshyn, The solution by iteration of nonlinear functional equations in Banach spaces, Bull. Amer. Math. Soc. 72 (1966), 571-575.

4. R. E. Bruck, W. A. Kirk, and S. Reich, Strong and weak convergence theorems for locally nonexpansive mappings in Banach spaces, Nonlinear Anal. TMA 6 (1982), 151-155.

5. K. Deimling, Zeros of accretive operators, Manuscripta Math. 13 (1974), 365-374.

6. T. Kato, Nonlinear semigroups and evolution equations, J. Math. Soc. Japan 19 (1967), 508-520.

7. W. A. Kirk, A fixed point theorem for local pseudo-contractions in uniformly convex spaces, Manuscripta Math. 30 (1979), 89-102.

8. W. A. Kirk and C. H. Morales, On the approximation of fixed points of locally nonexpansive mappings, Canad. Math. Bull. 24 (1981), 441-445.

9. C. H. Morales, Pseudo-contractive mappings and the Leray-Schauder boundary condition, Comment. Math. Univ. Carolin. 20 (1979), 745-756.

10. (1981), 71-74.

11. __ Strong convergence theorems for pseudo-contractive mappings in Banach space, Houston J. Math. 16 (1990), 549-557.

Department of Mathematical Sciences, University of Alabama in Huntsville, Huntsville, Alabama 35899

E-mail address: MORALES@MATH.UAH.EDU

Department of Physics, University of Zimbabwe, Harare, Zimbabwe 